\title{
Primary ileo-anal pouch anastomosis in patients with acute ulcerative colitis
}

Jacek Hermann, Jacek Szmeja, Tomasz Kościński, Wiktor Meissner, Michał Drews

Department of General, Gastrointestinal and Endocrinological Surgery, Poznan University of Medical Sciences, Poland

Submitted: 5 February 2011

Accepted: 14 July 2011

Arch Med Sci 2013; 9, 2: 283-287

DOI: 10.5114 /aoms.2013.33175

Copyright $\odot 2013$ Termedia \& Banach

\begin{abstract}
Introduction: Proctocolectomy with ileal pouch-anal anastomosis (IPAA) was performed in ulcerative colitis (UC) for emergent or urgent indications in three stages. Since the three-step procedure imposes enormous demands on a patient, there was an attempt to introduce primary IPAA for urgent indications. The aim of this study was to compare early complications after Hartmann's colectomy (HC) and IPAA in a selected group of patients.

Material and methods: Medical records of 274 patients who underwent surgery for UC between 1996 and 2010 were retrospectively evaluated. Finally, a group of 77 patients with acute form of UC entered this study.

Results: All patients were divided into two groups. Group 1 consisted of $32(42 \%)$ patients who underwent HC, whereas group 2 comprised 45 (58\%) patients after IPAA. There was no postoperative mortality. Respiratory failure occurred in $8(24 \%)$ patients after $\mathrm{HC}$ and in $6(14 \%)$ patients who underwent IPAA. Intraabdominal sepsis developed in 4 (12\%) patients after $\mathrm{HC}$ and in $8(17 \%)$ undergoing IPAA. Fascia dehiscence was present in $3(8 \%)$ patients after $\mathrm{HC}$ and in $4(9 \%)$ with IPAA. Bowel obstruction occurred in $1(4 \%)$ patient after the former operation and in $3(6 \%)$ patients after the latter one. Wound infection was diagnosed in $6(20 \%)$ patients after HC and in $9(20 \%)$ after IPAA. The differences between the investigated groups of patients were not statistically significant. Conclusions: The IPAA could be performed for urgent indications only in the patients with no critical dilatation of the colon or with active UC but without signs of severe malnutrition.
\end{abstract}

Key words: acute ulcerative colitis, urgent indications for surgery, Hartmann's colectomy, restorative proctocolectomy, early complications.

\section{Introduction}

There is a heterogeneous population of patients with active ulcerative colitis who require urgent surgery. They can be divided into two groups. Group 1 consists of patients who develop toxic megacolon with critical dilatation, severely malnourished patients with active ulcerative colitis (UC) unresponsive to medical treatment and those with intraoperative findings such as impending perforation or walled-off perforation. Group 2 includes patients with incipient toxic megacolon and patients with acute UC unresponsive to medical treatment with continuous or frequently relapsing form of exacerbation but without signs of critical malnutrition [1, 2]. Surgical treatment of acute UC follows intensive intravenous management including highdose steroids, parenteral nutrition, antibiotics, correction of electrolyte

\author{
Corresponding author: \\ Jacek Hermann PhD \\ Department of General \\ Gastrointestinal and \\ Endocrinological Surgery \\ Poznan University \\ of Medical Sciences \\ 49 Przybyszewski St \\ 60-355 Poznan, Poland \\ Phone: + 48618691275 \\ Fax: 48618691684 \\ E-mail: \\ jacekhermann@gmail.com
}


abnormalities and anemia. Although intense medical treatment breaks an acute attack of the disease in over half of the patients, relapse within the first year is common and approaches 50\% [3-5]. Restorative proctocolectomy with ileal pouch-anal anastomosis (IPAA) has become the procedure of choice in UC. It is a radical method and it provides the patients with good quality of life due to preserving anal sphincters, and therefore, fecal continence $[6,7]$. lleal pouch-anal anastomosis can be performed either in three or two stages. Three-step IPAA starts with Hartmann's colectomy followed by resection of the rectum and ileal "J-pouch" anal anastomosis with diverting loop-ileostomy and finally ileostomy closure, whereas the two-stage procedure consists of primary IPAA with loop-ileostomy and closure of that ileostomy as a final step [8-10]. In the past, threestage surgical treatment was recommended for all patients with urgent indications. It is unquestionable to start the treatment with Hartmann's colectomy $(\mathrm{HC})$ in the most severely ill patients with impending perforation, critical dilation of the large bowel and in whom correction of all abnormalities is difficult before the operation. However, because the threestage procedure puts enormous demands on the patients, adds another laparotomy to the management and prolongs the treatment, there has been an attempt to introduce primary pouch anal anastomosis with loop-ileostomy for urgent indications in the patients in a better general condition from group 2 $[11,12]$.

The aim of this study was to compare the patients after Hartmann's procedure with those undergoing primary restorative proctocolectomy for selected urgent indications in UC with regard to early complications based on the authors' experience and the literature.

\section{Material and methods}

Medical records of 274 patients who underwent surgery for UC at the Department of General, Gastrointestinal and Endocrinological Surgery in Poznan between 1996 and 2010 were retrospectively evaluated. Only patients with UC were included in this study. Diagnosis of UC was established using clinical, radiological, endoscopic and histopathological criteria. The extent of large bowel inflammation was determined on the basis of colonoscopy and/or barium enema. The degree of illness was defined according to modified Truelove and Witts' disease severity index taking into account clinical parameters on admission to hospital and before surgery $[1,3]$. Ninety-nine (36\%) patients underwent operations for emergent and urgent indications, whereas an elective procedure was done in 175 (64\%) patients. The operations were classified as emergent in cases of massive hemorrhage and colonic perforation or urgent in toxic megacolon and in acute attacks of UC unresponsive to intense medical treatment. Finally, the study encompassed $77 \mathrm{pa}-$ tients operated on for incipient form of toxic megacolon and for acute UC unresponsive to medical treatment with continuous or frequently relapsing form of exacerbation but without signs of critical malnutrition. The group of 22 "higher risk" patients with emergent indications and those with toxic megacolon with critical dilatation, severely malnourished patients with active UC unresponsive to medical treatment and those with intraoperative findings such as impending perforation or walled-off perforation were excluded from this study. The number of bowel movements with blood exceeded 6 , body temperature hovered between $37^{\circ} \mathrm{C}$ and $38^{\circ} \mathrm{C}$, blood pressure was over $100 \mathrm{~mm} \mathrm{Hg}$ and the pulse rate fluctuated from 90 to 110 beats per minute. Hemoglobin level less than $10 \mathrm{~g} / \mathrm{dl}$ was corrected with transfusions before operations. All the patients included in this study were given steroids before the operation. The average dose of methylprednisolone did not exceed $16 \mathrm{mg}$ on the day of operation in 40 patients operated on for exacerbation of UC after aggressive tapering started just after admission to hospital. The dose of hydrocortisone on the day of operation averaged $200 \mathrm{mg}$ in the remaining patients. Medical records were evaluated with regard to sex, age and the number of patients, duration of disease, indications for surgery, surgical methods and early postoperative complications. The choice of operation was determined by severity of illness as well as the surgeon's preference.

\section{Statistical analysis}

The results were evaluated statistically. The complication rates were compared with the test for the differences between the two proportions and the differences accepted as non-significant at $p>0.05$.

\section{Results}

There were 99 patients who underwent surgery for emergent or urgent indications. Finally, 77 patients with a severe attack of UC entered the study after exclusion of 22 higher-risk patients. There were 40 (52\%) males and 37 (48\%) females. The mean age was 38 years (range: $14-72$ years). Median duration of disease until primary operation was 3 years (range: 4 weeks to 20 years). All the patients were divided into two groups according to the operation method. Group 1 consisted of 32 patients who underwent HC, whereas group 2 comprised 45 patients after ileal pouch-anal anastomosis. Primary acute attack of UC unresponsive to medical treatment was an indication for surgery in 15 patients, 9 patients underwent an operation due to continuous form of acute UC and 44 (57\%) patients were referred to surgery because of exacerbation of chronic form of UC. Hartmann's operation was 
performed in 5 and IPAA in 4 patients due to incipient toxic megacolon. There were no postoperative deaths in the surveyed group of patients. Respiratory failure occurred in $8(24 \%)$ patients after Hartmann's operation and in 6 (14\%) patients who underwent the pouch procedure and it was the most frequent and severe general complication. Intra-abdominal sepsis was the most serious surgical complication. That complication developed in $4(12 \%)$ patients after Hartmann's colectomy in the form of minor pelvis abscess in 3 patients and multiple intra-abdominal abscesses in 1 patient. Similar infection occurred in $8(17 \%)$ patients after IPAA and it developed in the form of minor pelvis abscess in 4 patients, diffuse peritonitis in 2 and multiple intraabdominal abscesses also in 2 patients. Most patients with intra-abdominal sepsis required surgery except for 2 patients after $\mathrm{HC}$ and 3 patients after IPAA who developed small minor pelvis abscesses and were treated with percutaneous drainage. Fascia dehiscence was present in 3 (8\%) patients undergoing $\mathrm{HC}$ and in 4 (9\%) after the pouch procedure. Bowel obstruction treated with surgical measures occurred in 1 patient after the former operation and in $3(6 \%)$ patients after the latter one. Wound infection, the most frequent surgical complication, was diagnosed in $6(20 \%)$ patients after colectomy and in $9(20 \%)$ after proctocolectomy. The differences between the investigated groups of patients were not statistically significant (Table I).

\section{Discussion}

Three-step restorative proctocolectomy with ileal "J-pouch" anal anastomosis remains unquestionably the operation of choice in the treatment of patients with acute UC for emergent and for some patients with urgent indications. The first step, which is $\mathrm{HC}$ with end ileostomy, is indicated in severely ill patients due to massive lower digestive tract bleeding, diffuse fecal peritonitis from colonic perforation, fragile tissues due to inflammation and severe inflammatory infiltration within the rectum and mesorectum and for clinical deterioration with progressive dilation in the course of toxic megacolon. The aim of that procedure is to remove the diseased bowel while decreasing the morbidity associated with rectum mobilization and bowel anastomosis [10, 13-15]. However, there has been a shift regarding indications for surgery within the past decades from a decreasing number of patients with toxic megacolon and perforation to an increasing rate of patients with acute colitis unresponsive to intense medical treatment $[16,17]$. As a result, there is a significant group of patients operated on for acute UC who could undergo primary IPAA with loop-ileostomy that is a two-step treatment and who might profit from such management by avoiding another large abdominal operation and potential serious complications. These are patients in the acute phase of UC who fail to respond to medical treatment, have no signs of perforation or critical dilation of the colon, and do not show signs of critical malnutrition. The investigated group of patients who matched the aforementioned criteria underwent either $\mathrm{HC}$ or primary IPAA. The morbidity rate after either Hartmann's procedure or pouch surgery occurring in this study was high. However, the rate of complications did not exceed the frequency of complications reported from other centers [16, 18-21]. In addition, the rate of early complications between the investigated groups of patients was not statistically significant. Intraabdominal sepsis was the most serious surgical complication in this survey. Failure to react in a timely fashion could lead to poor pouch function. Abdominal infection could arise due to either dehiscence of Hartmann's pouch or ileal-pouch anal anastomosis despite performing loop-ileostomy. latrogenic bowel perforation and infected pelvic hematoma as a result of translocation of bacteria from the diseased colon should also be taken into account as a possible cause of surgical site infection. This complication appeared as either circumscribed peritonitis in the form of minor pelvis abscess and multiple intraabdominal abscesses or as diffuse peritonitis. Other authors also reported pelvic sepsis in the form of pouch fistulae. Digital examination may reveal an anastomotic defect or localized tenderness overlying an indurated or fluctuant mass. Computed tomography is the procedure of choice to demonstrate its extent. All the complications were cured though

Table I. Early complications after urgent operations for acute ulcerative colitis during 1996-2010

\begin{tabular}{|lccc|}
\hline Complication & $\begin{array}{c}\text { Hartmann's } \\
\text { colectomy }(n=32)\end{array}$ & $\begin{array}{c}\text { Proctocolectomy with ileo-anal } \\
\text { pouch anastomosis }(n=45)\end{array}$ & Value of $p$ \\
\hline Wound infection, $n(\%)$ & $6(20)$ & $9(20)$ & 0.892 \\
\hline Intra-abdominal sepsis, $n(\%)$ & $4(12)$ & $8(17)$ & 0.531 \\
\hline Fascia dehiscence, $n(\%)$ & $3(8)$ & $4(9)$ & 0.942 \\
\hline Bowel obstruction, $n(\%)$ & $1(4)$ & $3(6)$ & 0.492 \\
\hline Respiratory failure, $n(\%)$ & $8(24)$ & $6(14)$ & 0.195 \\
\hline Morbidity, $n(\%)$ & $22(69)$ & $30(67)$ & 0.848 \\
\hline
\end{tabular}

Complication rates were compared with the test for differences between two proportions and the differences accepted as non-significant at $p>0.05$ 
there was a need for another surgical intervention in most cases. Although small abscesses were treated with broad spectrum antibiotics, bigger ones required transanal guided drainage. Re-laparotomy was indicated for multiple abscesses, diffuse peritonitis and collections, if guided drainage failed to control sepsis [22, 23]. Some authors advise drainage and irrigation of both Hartmann's and ileal pouches to remove residual blood and mucus, which could be a source of infection and the cause of pelvic sepsis [21]. Others incorporate a long Hartmann's stump in the fascial closure which results in wound infection or a mucous fistula rather than in pelvic abscess [24]. Some authors have found a decreased rate of pelvic abscess when the rectal stump was exteriorized [21]. Other surgeons have reported a low rate of pelvic sepsis with an intraperitoneal stump and they advocate routine transanal drainage of the rectum [25]. It has been well established that the composition of gut microflora in UC patients changes to increased pathogenic bacteria and decreased bifidobacteria and lactobacilli. Such dysregulation between aggressive and beneficial bacterial species could result in exacerbation of any intra-abdominal sepsis. Therefore, administration of probiotics in UC patients could alleviate possible postoperative septic complications [26, 27]. However, poor general condition of seriously ill, immunocompromised, malnourished patients suffering from acute UC is the major cause of increased morbidity and increased susceptibility to infections. Therefore, primary IPAA is accepted only in a selected group of patients in whom most abnormalities can be corrected preoperatively. Such management consists of anemia and water-electrolyte abnormalities correction, antibiotics, early introduction of intense parenteral nutrition and its continuation despite restoration of oral nutrition after the operation to correct malnutrition and aggressive tapering of steroids within a few days after the operation, although one must bear in mind the risk of adrenal insufficiency. Steroids could either impair healing of any anastomosis, decrease the ability to combat infection or simply mark a group of patients in poor general condition. However, some authors argue that the dose of hydrocortisone exceeding $200 \mathrm{mg}$ before the operation and leukocytosis over $10 \times 10^{9} / \mathrm{l}$ are independent risk factors of anastomosis dehiscence. Therefore, patients on a high dose of steroids should undergo preliminary colectomy with rectal preservation instead of a primary ileo-anal pouch procedure [11, 12, 25, 28].

Finally, successful treatment of chronic, extensive and intractable UC can be achieved with earlier referral to surgery of patients in remission rather than treatment of those in the acute phase of the disease [29].

In conclusion, primary restorative proctocolectomy with ileostomy could be performed for urgent indications only in a selected group of patients such as those with no critical dilatation of the colon or with active UC unresponsive to medical treatment with continuous or frequently relapsing form of exacerbation but without signs of severe malnutrition.

\section{References}

1. Truelove SC. Systemic and local corticosteroid therapy in ulcerative colitis. Br Med J 1960; 13: 464-73.

2. Berg DF, Bahadursingh AM, Kaminski DL, Longo WE. Acute surgical emergencies in inflammatory bowel disease. Am J Surg 2002; 184: 45-51.

3. Truelove SC. Medical management of ulcerative colitis and indications for colectomy. World J Surg 1988; 12: 142-7.

4. Jewell DP. Medical management of severe ulcerative colitis. Int J Colorectal Dis 1988; 3: 186-9.

5. Katz JA. Medical and surgical management of severe colitis. Semin Gastrointest Dis 2000; 11: 18-32.

6. Williams NS. Restorative proctocolectomy is the first choice elective surgical treatment for ulcerative colitis. Br J Surg 1989; 76: 1109-10.

7. Galandiuk S, Scott NA, Dozois RR, et al. Ileal pouch-anal anastomosis. Ann Surg 1990; 212: 4: 446-52.

8. Kelly KA, Pemberton JH, Wolff BG, Dozois RR. Ileal pouchanal anastomosis. Curr Probl Surg 1992; 29: 61-131.

9. Hwang JM, Varma MG. Surgery for inflammatory bowel disease. World J Gastroenterol 2008; 14: 2678-90.

10. Hyman NH, Cataldo P, Osler T. Urgent subtotal colectomy for severe inflammatory bowel disease. Dis Col Rectum 2004; 48: 70-3.

11. Harms BA, Myers GA, Rosenfeld DJ, Starling JR. Management of fulminant ulcerative colitis by primary restorative proctocolectomy. Dis Col Rectum 1994; 37: 971-8.

12. Ziv Y, Fazio VW, Church JM, Milsom JW, Schroeder TK. Safety of urgent restorative proctocolectomy with ileal pouchanal anastomosis for fulminant colitis. Dis Col Rectum 1995; 38: 345-9.

13. Crile GC, Thomas CY. The treatment of acute toxic ulcerative colitis by ileostomy and simultaneous colectomy. Gastroenterology 1951; 19: 58-68.

14. Goligher JC, Hoffman DC, de Dombal FT. Surgical treatment of severe attacks of ulcerative colitis with special reference to the advantages of early operation. Br Med J 1970; 4: 703-6.

15. Hawley PR. Emergency surgery for ulcerative colitis. World J Surg 1988; 12: 169-73.

16. Teeuwen PHE, Stommel MWJ, Bremers AJA, van der Wilt GJ, de Jong DJ, Bleichrodt RP. Colectomy in patients with acute colitis: a systematic review. J Gastrointest Surg 2009; 13: 676-86.

17. Chebli LA, Felga GGE, Miranda Chaves LD, et al. Early onset steroid-dependent ulcerative colitis is a predictor of Azathioprine response: a longitudinal 12-month follow-up study. Med Sci Monit 2010; 16: 1-6.

18. Fazio VW, Ziv Y, Church JM. Ileal pouch-anal anastomosis complications and function in 1005 patients. Ann Surg 1995; 222: 120-7.

19. Binder SC, Miller HH, Deterling RA. Emergency and urgent operations for ulcerative colitis. Arch Surg 1975; 110: 284-9.

20. Albrechtsen D, Bergan A, Nygaard K. Urgent surgery for ulcerative colitis: early colectomy in 132 patients. World J Surg 1981; 5: 607-15.

21. Carter FM, McLeod RS, Cohen Z. Subtotal colectomy for ulcerative colitis: complications related to the rectal remnant. Dis Col Rectum 1991; 34: 1005-9.

22. Farouk R, Dozois RR, Pemberton JH. Incidence and subsequent impact of pelvic abscess after ileal pouch-anal 
anastomosis for chronic ulcerative colitis. Dis Colon Rectum 1998; 41: 1239-43.

23. Heuschen UA, Allemeyer EH, Hinz U, Lucas M, Herfarth C, Heuschen G. Outcome after septic complications in J pouch procedures. Br J Surg 2002; 89: 194-200.

24. Trickett JP, Tilney HS, Gudgeon AM. Management of the rectal stump after emergency subtotal colectomy: which surgical option is associated with the lowest morbidity? Colorectal Dis 2005; 7: 519-22.

25. Karch LA, Bauer JJ, Gorfine SR, Gelernt IM. Subtotal colectomy with Hartmann`s pouch for inflammatory bowel disease. Dis Colon Rectum 1995; 38: 635-9.

26. Rahimi R, Nikfar S, Rezaie A, Abdollahi M. A meta-analysis of benefit of probiotics in maintaining remission of human ulcerative colitis: evidence for prevention of disease relapse and maintenance of remission. Arch Med Sci 2008; 4: 185-90.

27. Nikfar S, Darvish-Damavandi M, Abdollahi M. A review and meta-analysis of the efficacy of antibiotics and probiotics in management of pouchitis. Int J Pharmacol 2010; 6: 826-35.

28. Fazio VW, Tekkis PP, Remzi F. Quantification of risk for pouch failure after ileal pouch anal anastomosis surgery. Ann Surg 2003; 238: 605-14.

29. Heyvaert G, Penninckx F, Filez L, Aerts R, Kerremans R, Rutgeerts P. Restorative proctocolectomy in elective and emergency cases of ulcerative colitis. Int J Colorectal Dis 1994; 9: 73-6. 